\title{
The magnitude of dexamethasone-induced hyperglycemia in cataract surgical patients with normal glucose metabolism: a continuous glucose monitoring based study
}

\author{
Wen-li Zhang \\ Gu Gao \\ Xiao-juan Sun \\ Lei Ye \\ Jian-hua Ma \\ Feng-fei Li ( $\square$ lifengfei2005@126.com ) \\ https://orcid.org/0000-0002-6127-6604
}

\section{Research}

Keywords: dexamethasone, hyperglycemia, normal glucose metabolism

Posted Date: February 11th, 2020

DOl: https://doi.org/10.21203/rs.2.23145/v1

License: (c) (i) This work is licensed under a Creative Commons Attribution 4.0 International License. Read Full License 


\section{Abstract}

Background To determined 24-hrs glycemic variations using continuous glucose monitoring (CGM) in cataract surgical patients with normal glucose metabolism receiving dexamethasone therapy.

Methods This was a single-center, randomized, observational and prospective trial. Between June 2017 and December 2018, cataract surgical patients with normal glucose toleration were recruited, and were randomized assigned at a ratio of 1:2 to either receiving $10 \mathrm{mg}$ dexamethasone daily for 3 days after cataract surgical (dexamethasone group) or without dexamethasone therapy (control group). A retrospective CGM was performed at afternoon on -1 day before cataract operation performed for at least 3 days. The primary endpoint was the difference in mean amplitude of glycemic excursions (MAGE) between the two groups.

Results We observed that patients receiving $10 \mathrm{mg}$ dexamethasone daily showing mild increase in mean glucose and hourly mean glucose throughout 24-hrs compared to control group. We also detected that dexamethasone leading to a significant increase in glycemic variation, in term of standard deviation, and a number increase in MAGE and CV\%.

Conclusion Our data reveal that short period administration of dexamethasone leading a mild increase in glycemic variations (GV). However, the increased GV may have not clinically relevant. Our data indicates that short duration of $10 \mathrm{mg}$ dexamethasone once daily may be a safe therapy regarding glycemic metabolism in subjects without diabetes.

\section{Background}

Evidence indicates that above $10 \%$ inpatients need steroid for variety disease[1], with the treatment duration vary from less than 5 days to $>5$ years [2]. An impairment of glycemic metabolism or even steroid-induced diabetes may occur in subjects with normal glucose tolerate [3]. Although a pilot study observed that the use of high-dose dexamethasone may not lead to glucocorticoid-induced diabetes [4]. Using fasting and 2-hour postprandial glucose levels, and hemoglobin $\mathrm{A}_{1 \mathrm{C}}$ values, Jeong $\mathrm{Y}$ et al. observed that non-diabetic patients had a maximum of $20 \%$ risk ratio of development of diabetes at 3 or 6 months after dexamethasone therapy [5]. Furthermore, the higher incidence of glucocorticoid induced diabetes may significantly decrease after 30 months[6]. The magnitude and duration of dexamethasone induced glucose level increase may be mild and transient in patients without diabetes[7, 8]. Importantly, researchers observed that patients without prior history of diabetes showing a comparable [9], or greater increase in glucose levels compared to those of patients with onset diabetes [10]. Furthermore, patients received $>13.5 \mathrm{mg}$ dexamethasone per day ( 5 days) had an increase incidence of hyperglycemia than those who received other types of steroids[11]. However, available evidences regarding the glycemic response to dexamethasone based on the time point of glucose readings, which weaken the findings on glycemic variations (GV) throughout 24 hours. 
Continuous Glucose Monitoring (CGM) provides glucose readings each 15 min lasting for at least 3 days, which is a unique technique to assess the GV throughout a day in inpatients receiving dexamethasone therapy. We therefore performed a single-center, randomized, observational and prospective study. In this study, we determined 24-hrs glycemic variations using CGM in cataract surgical patients with normal glucose metabolism receiving dexamethasone therapy.

\section{Methods}

This was a single-center, randomized, observational and prospective trial. Between June 2017 and December 2018, cataract surgical patients with normal glucose toleration were recruited in Nanjing First Hospital, Nanjing Medical University, China. The inclusion criteria were 1) Inpatients aged between 18 and 80 years; 2) with normal glucose metabolism, defined either by fasting glucose or by $75 \mathrm{~g} \mathrm{OGTT;3)} \mathrm{BMI}$ 18 to $35 \mathrm{~kg} / \mathrm{m}^{2}$. Patients were excluded if they had known cancers $[12,13]$. The study was approved by the ethics committee of Nanjing First Hospital, Nanjing Medical University. All patients gave written informed consent. The methods were conducted in accordance with the Declaration of Helsinki guidelines, including any relevant details.

All enrolled subjects were admitted as inpatients, and were randomized assigned at a ratio of 1:2 to either receiving $10 \mathrm{mg}$ dexamethasone daily for 3 days after cataract surgical (dexamethasone group) or without dexamethasone therapy (control group). At baseline, demographic characteristics were collected, and serum samples were obtained at 0,30 , and $120 \mathrm{~min}$ after oral administration of $75 \mathrm{~g}$ glucose diluted in $200 \mathrm{ml}$ water for glucose, and C-peptide levels determination. Plasma $\mathrm{HbA}_{1 \mathrm{c}}$ was measured by a DiaSTAT $\mathrm{HbA}_{1 \mathrm{c}}$ analyzer (Bio-Rad, Hercules, CA). C-peptide and glucose concentrations were determined centrally at the central laboratory in Nanjing First Hospital, Nanjing Medical University. A retrospective CGM (Sof-sensor, CGMS-Gold, Medtronic Incorporated, Northridge, USA) was performed at afternoon on -1 day before cataract operation performed for at least 3 days, as described previously $[14,15]$. Briefly, the CGM sensor was subcutaneously fixed on Day -1 at 16:00-17:00 PM by specially trained research nurses. The patients continued with the sensors for at least 3 days, and at least 4 calibration readings were inputted daily during the CGM period. The CGM data were saved by the investigator, as described previously [14-16]. During the CGM period, all patients were maintained physical activity according to their doctors' personalized instruction. Patients were served three meals at 0700, 1100 and 1700, with a total daily caloric intake of $25 \mathrm{kcal} / \mathrm{kg} /$ day containing carbohydrate, proteins and fats were $55 \%, 17 \%$ and $28 \%$, respectively. In addition, at least four blood glucose readings were recorded by the study nurses. The glucose profiles delivered from CGM in terms of the 24-hrs MG, the SDBG, the hourly MG, and the MAGE were calculated by software (Medtronic Incorporated, USA) or by manually, as described previously [14, 15].

The primary endpoint was the difference in mean amplitude of glycemic excursions (MAGE) between the two groups. Secondary endpoints were the differences in 24-hrs mean glucose (MG), the standard deviation of MG (SDMG), the coefficient of variation (CV\%), and hourly MG levels. 


\section{Results}

\section{Demographic profiles}

From June 2017 to December 2018, a total of 30 cataract surgical patients with normal glucose toleration were recruited in Nanjing First Hospital, Nanjing Medical University, China, and all patients completed this study. Consecutively recruited subjects (who received cataract surgical) were randomized assigned to dexamethasone group $(n=20)$ and control group $(n=10)$. There were no significant demographic differences between groups at baseline. Also, there were no differences in $\mathrm{HbA}_{1 \mathrm{c}}$, mean 0 , 30 , and 120 min blood glucose, and C-Peptide levels after glucose loading between the two groups (Table 1).

Table 1

Characteristics in recruited patients at baseline between two groups

\begin{tabular}{|llll|}
\hline Items & Dexamethasone group & Control group & P \\
\hline $\mathrm{N}$ & 22 & 10 & $/$ \\
Sex & Male 11 & Male 5 & 1.000 \\
Age & $50.55 \pm 18.01$ & $57.90 \pm 17.25$ & 0.287 \\
$\mathrm{BMI}\left(\mathrm{Kg} / \mathrm{m}^{2}\right)$ & $22.49 \pm 3.19$ & $22.88 \pm 2.62$ & 0.740 \\
\hline HbA1c $(\%)$ & $5.37 \pm 0.35$ & $5.34 \pm 0.34$ & 0.833 \\
Glu 0 & $4.85 \pm 0.47$ & $5.01 \pm 0.24$ & 0.330 \\
Glu120 & $5.82 \pm 1.02$ & $6.82 \pm 0.44$ & 0.001 \\
\hline \multicolumn{4}{|l}{ N, number, BMl: Body mass index, Glu 0: Fasting plasma glucose (mmol/L), Glu 120: the blood } \\
glucose level after glucose loading 120 minutes (mmol/L). \\
\hline
\end{tabular}

Table 2 Changes in blood glycemic variation parameters in patients between the two groups

\begin{tabular}{lccc}
\hline & Dexamethasone group & Control group & P value \\
\hline 24-hrs MBG & $8.23 \pm 1.27$ & $6.01 \pm 0.38$ & 0.001 \\
SD & $1.37 \pm 0.77$ & $0.95 \pm 0.34$ & 0.040 \\
\hline MAGE & $3.12 \pm 1.94$ & $2.16 \pm 0.88$ & 0.066 \\
\hline CV\% & $0.16 \pm 0.08$ & $0.16 \pm 0.06$ & 0.055 \\
\hline
\end{tabular}

24-hrs MBG: 24h mean blood glucose (mmol/L), SD: Standard deviation for the mean glucose concentration (mmol/L), MAGE: mean amplitude of glycemic excursions (mmol/L), CV\%: coefficient of variation \%.

\section{Discussion}

In this observational study, we observed that patients receiving $10 \mathrm{mg}$ dexamethasone daily for 3 days showing mild increase in MBG and hourly glucose readings throughout 24-hrs compared those who control group. Our CGM data also indicated that dexamethasone leading to a significant increase in GV, in 
term of SD, and a number increase in MAGE and CV\%. Our findings indicated that subjects with NGT receiving $10 \mathrm{mg}$ for a short period may be a safe therapy regarding glycemic metabolism.

The risk-benefit ratio in patients without diabetes is debate issue for a long time. In this study, we employed CGM to assess the 24-hrs glycemic variations in cataract surgical patients with normal glucose tolerance receiving $10 \mathrm{mg}$ once daily for 5 days. Our data showed that the MBG and SD were increased in dexamethasone group compared to those of control group, with a $2.22 \mathrm{mmol} / \mathrm{L}$ increase in glucose levels and $0.42 \mathrm{mmol} / \mathrm{L}$ in SD. However, the increase in glucose concentrations and GV may have not clinically relevant. Because according to the glucose readings delivered from CGM, subjects receiving dexamethasone exhibited the maximum glucose level was less than $11.1 \mathrm{mmo} / \mathrm{L}$, and the SD less than $1.4 \mathrm{mmol} / \mathrm{L}[17]$. Our results in accordance with a previous study reporting that patients receiving high dose of dexamethasone did not resulting in diabetes, and the rise in glucose concentrations were transient and improvement after 6 weeks follow up[4]. In contrast to our study, previously published data showed that about one fifth non-diabetic patients receiving dexamethasone therapy were prone to development of diabetes after long-run follow up [5]. The differences in corticosteroid associated hyperglycemia between the two study may be our study with a relatively short follow up period, because of exposure to a large cumulative dose of dexamethasone may impair glycemic metabolisms[4].

A strength of our study was we employed CGM for determine the 24-hrs glucose profiles of patients throughout the dexamethasone period, which providing a set of metrics describing the GV in patients with or without of diabetes [18-20]. Using CGM data, we analyzed GV, in terms of MAGE, SD, and CV\%, between the two groups. We observed a significantly increase in SD in patients receiving dexamethasone therapy compared to those of control group. Although the values of MAGE between the two groups failed to achieve statistically Although researchers indicate that there was a high degree of correlation between SD and MAGE $[21,22]$. We only observed a discernable increase in MAGE values in patients receiving dexamethasone therapy, which may be the modest sample size in this study. Another strength of this study should also be addressed. To identify the rise in glucose level was secondary to the dexamethasone therapy or to the surgical stress response, we analyzed CGM data from who received cataract surgical plus dexamethasone administration and who received cataract surgical only. Our data showed that dexamethasone had the extra glucose-increasing effect beyond surgical stress response, which may add information back up a previous study lacking control group reporting that patients showing increases in blood glucose levels after administration of dexamethasone[9].

Corticosteroid associated hyperglycemia may have a pronounced peak glucose concentration after 9 to 12 hours [8, 23], and gradually decrease to baseline or to normal after nocturnal period [11]. However, in this study, we did not observe a discernable trend of glycemic decrease from afternoon to next morning. A reasonable explanation might be the relatively modest subjects were enrolled, we have now address this as another limitation of this study.

\section{Conclusion}


In conclusion, our data reveal that short period administration of dexamethasone leading a mild increase in glycemic variations (GV). However, the increased GV may have not clinically relevant. Our data indicates that short duration of $10 \mathrm{mg}$ dexamethasone once daily may be a safe therapy regarding glycemic metabolism in subjects without prior diabetes.

\section{Declarations}

\section{Ethics approval and consent to participate}

The study was approved by the ethics committee of Nanjing First Hospital, Nanjing Medical University. All patients gave written informed consent. The methods were conducted in accordance with the Declaration of Helsinki guidelines, including any relevant details.

\section{Consent for publication}

Written informed consent for publication was obtained from all participants.

\section{Data availability statement}

The datasets generated during and/or analyzed during the current study are available from the corresponding author on reasonable request.

\section{Competing interests}

No competing financial interests exist.

\section{Funding}

This research was funded by the National Key R\&D Program of China (2018YFC1314100), the Nanjing Municipal Science and Technology Project (No. 201803011), the Science and Technology Development Foundation of Nanjing Medical University (No. 2017NJMUZD108, and 2017NJMU128).

\section{Authors' Contributions Statement}

J.M., and FF. L. contributed to the conception and design of the study. FF. L., G. G., XJ. S. contributed to the conduct/data collection. G. G., and WL. Z. contributed to the data analysis. FF. L., G. G. contributed to writing the manuscript and the final approval of the manuscript.

\section{Acknowledgments}

We appreciated Prof. Lei Ye who contributed to the final proof of the manuscript. 


\section{References}

1. Narwani, V., et al., How frequently are bedside glucose levels measured in hospital inpatients on glucocorticoid treatment? Clin Med (Lond), 2014. 14(3): p. 327-8.

2. Fardet, L., I. Petersen, and I. Nazareth, Prevalence of long-term oral glucocorticoid prescriptions in the UK over the past 20 years. Rheumatology (Oxford), 2011. 50(11): p. 1982-90.

3. Roberts, A., J. James, and K. Dhatariya, Management of hyperglycaemia and steroid (glucocorticoid) therapy: a guideline from the Joint British Diabetes Societies (JBDS) for Inpatient Care group. Diabet Med, 2018. 35(8): p. 1011-1017.

4. Alabbood, M., M. Ling, and K. Ho, Effect of high-dose dexamethasone on patients without diabetes during elective neurosurgery: a prospective study. Diabetol Int, 2019. 10(2): p. 109-116.

5. Jeong, Y., et al., A Pilot Study Evaluating Steroid-Induced Diabetes after Antiemetic Dexamethasone Therapy in Chemotherapy-Treated Cancer Patients. Cancer Res Treat, 2016. 48(4): p. 1429-1437.

6. Fizazi, K., et al., Low Incidence of Corticosteroid-associated Adverse Events on Long-term Exposure to Low-dose Prednisone Given with Abiraterone Acetate to Patients with Metastatic Castration-resistant Prostate Cancer. Eur Urol, 2016. 70(3): p. 438-44.

7. Polderman, J.A., et al., Adverse side effects of dexamethasone in surgical patients. Cochrane Database Syst Rev, 2018. 8: p. CD011940.

8. Polderman, J.A.W., et al., Adverse sideeffects of dexamethasone in surgical patients - an abridged Cochrane systematic review. Anaesthesia, 2019. 74(7): p. 929-939.

9. Hans, $\mathrm{P}$. , et al., Blood glucose concentration profile after $10 \mathrm{mg}$ dexamethasone in non-diabetic and type 2 diabetic patients undergoing abdominal surgery. $\mathrm{Br} J$ Anaesth, 2006. 97(2): p. 164-70.

10. Abdelmalak, B.B., et al., The hyperglycemic response to major noncardiac surgery and the added effect of steroid administration in patients with and without diabetes. Anesth Analg, 2013. 116(5): p. 1116-22.

11. Fong, A.C. and N.W. Cheung, The high incidence of steroid-induced hyperglycaemia in hospital. Diabetes Res Clin Pract, 2013. 99(3): p. 277-80.

12. Ziegler, R., et al., ProAct study: new features of insulin pumps improve diabetes management and glycemic control in patients after transition of continuous subcutaneous insulin infusion systems. Diabetes Technol Ther, 2013. 15(9): p. 738-43.

13. Weng, J., et al., Effect of intensive insulin therapy on beta-cell function and glycaemic control in patients with newly diagnosed type 2 diabetes: a multicentre randomised parallel-group trial. Lancet, 2008. 371(9626): p. 1753-60.

14. Li, F.F., et al., Influence of Acarbose on Plasma Glucose Fluctuations in Insulin-Treated Patients with Type 2 Diabetes: A Pilot Study. Int J Endocrinol, 2015. 2015: p. 903524. 
15. Li, F.F., et al., Blood Glucose Fluctuations in Type 2 Diabetes Patients Treated with Multiple Daily Injections. J Diabetes Res, 2016. 2016: p. 1028945.

16. Zhou, J., et al., Reference values for continuous glucose monitoring in Chinese subjects. Diabetes Care, 2009. 32(7): p. 1188-93.

17. Zhou, J., et al., Establishment of normal reference ranges for glycemic variability in Chinese subjects using continuous glucose monitoring. Med Sci Monit, 2011. 17(1): p. CR9-13.

18. Fabris, C., et al., Glucose variability indices in type 1 diabetes: parsimonious set of indices revealed by sparse principal component analysis. Diabetes Technol Ther, 2014. 16(10): p. 644-52.

19. Fabris, C., et al., Parsimonious Description of Glucose Variability in Type 2 Diabetes by Sparse Principal Component Analysis. J Diabetes Sci Technol, 2015. 10(1): p. 119-24.

20. Rodbard, D., New and improved methods to characterize glycemic variability using continuous glucose monitoring. Diabetes Technol Ther, 2009. 11(9): p. 551-65.

21. Rodbard, D., et al., Improved quality of glycemic control and reduced glycemic variability with use of continuous glucose monitoring. Diabetes Technol Ther, 2009. 11(11): p. 717-23.

22. Rodbard, D., Increased glycemic variability at the onset and during progression of type 2 diabetescommentary. Diabetes Technol Ther, 2013. 15(6): p. 445-7.

23. Lukins, M.B. and P.H. Manninen, Hyperglycemia in patients administered dexamethasone for craniotomy. Anesth Analg, 2005. 100(4): p. 1129-33.

\section{Figures}




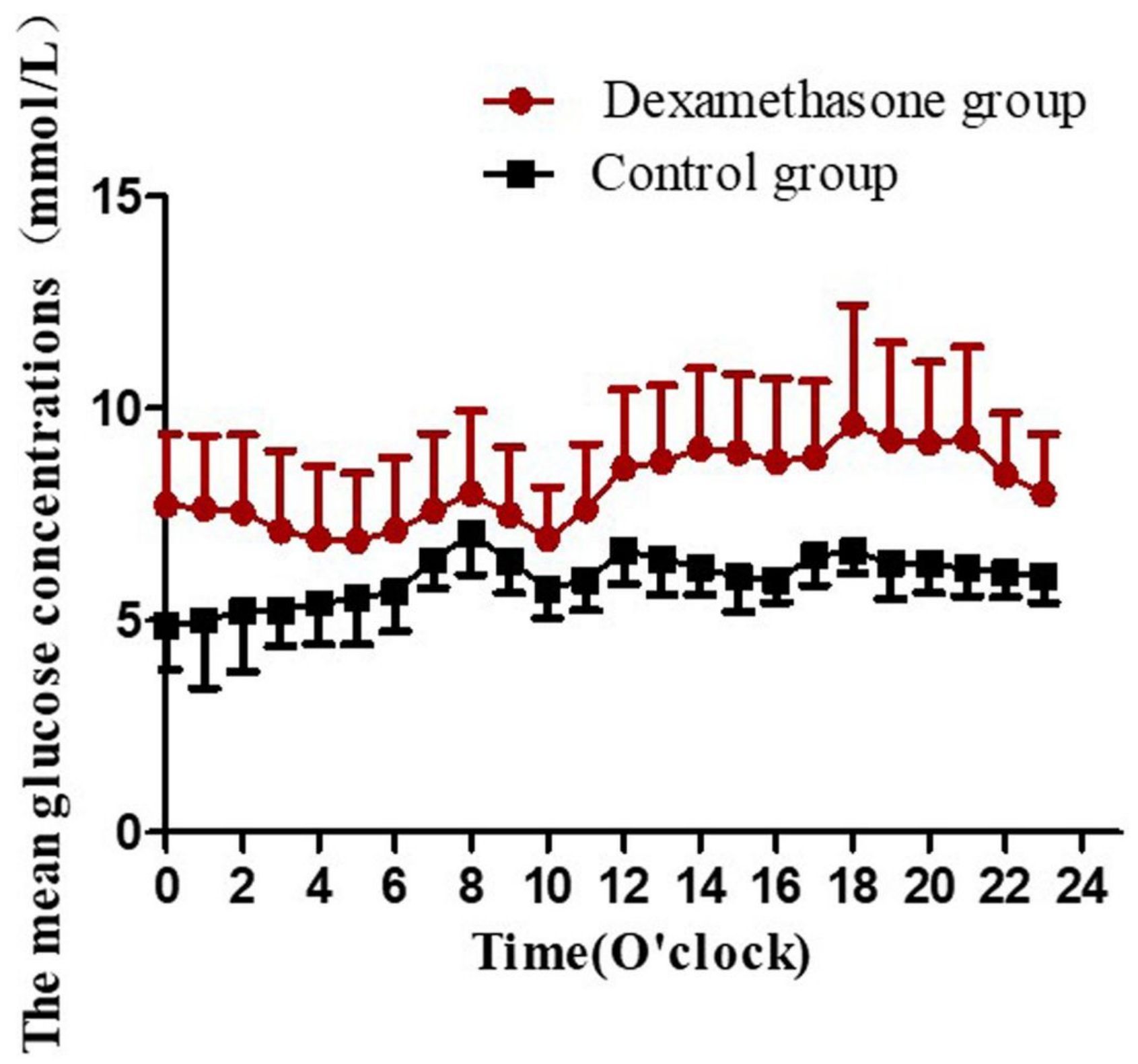

Figure 2

Hourly glucose levels between the two groups 